\title{
Web-Based Sensitivity Training for Interacting with Facial Paralysis
}

\author{
Zhang, Nicole (University of Warwick) \\ Michael, John (Central European University) \\ Bogart, Kathleen (Oregon State University) \\ McEllin, Luke (University of Warwick)
}

\begin{abstract}
:
Previous research has shown that observers tend to form inaccurate, negatively biased first impressions of people with facial paralysis (FP). This is likely to be due in part to limits which facial paralysis imposes upon the expression of information about emotional states. It has been hypothesised that this problem may be ameliorated by a training program designed to encourage people who will encounter individuals with FP to focus on other channels of expression rather than the face, e.g. hand gestures, body language, tone of voice and speech content. We tested this hypothesis in two web-based studies. In Study 1, participants in the Trained Condition received tips for understanding/interacting with individuals with FP, and practice in identifying emotions expressed through body language. Participants in the Untrained Condition received only general information about FP, and practice in identifying emotions expressed through facial expression. In the test phase, we compared the two groups' perception of emotions expressed in videos of individuals with FP, as well as their recall of the content of those videos. The results show that attending to bodily cues and to speech rather than facial cues can improve social perception and reduce bias. Study 2 tested participants in the Trained group two months later. The results show that the effects of the training did not persist. Taken together, our findings support the hypothesis that even brief training in attending to non-facial cues when interacting with individuals with FP can improve social perception and reduce bias, but that these effects do not persist over longer time periods in the absence of further training.
\end{abstract}

Keywords: facial paralysis, interpersonal sensitivity training, social perception, implicit bias 


\section{Introduction}

Previous research has shown that lay people as well as healthcare providers tend to form inaccurate first impressions of people with facial paralysis (FP) (Tickle-Degnen \& Lyons, 2004; Bogart, Tickle-Degnen, \& Ambady, 2014; Hemmesch, Tickle-Degnen, \& Zebrowitz, 2009). Moreover, they also exhibit a negative bias such that they tend to perceive people with FP as less extroverted and less happy than people without FP (Lyons et al., 2004). This may be due in part to limits which facial paralysis imposes upon the expression of information about emotional states (Krueger \& Michael, 2012).

To address this, Bogart \& Tickle-Degnen (2015) developed a brief set of training tips designed to encourage people who will encounter individuals with FP to focus on other channels of expression rather than the face, e.g. hand gestures, body language, tone of voice and speech content. Their results provide preliminary evidence that such a training program can reduce negative bias towards people with FP.

Building on this previous finding, we developed a training program consisting of the same set of tips employed in Bogart \& Tickle-Degnen's study, but also including a brief training phase in which participants in the training condition practiced identifying emotional expressions in body language. We implemented the program online, using prolific academic to recruit participants from the general population.

To test the efficacy of the training program, we also administered a series of tasks on which we compared the performance of those participants who had received the training (i.e. in the Trained Condition) with a separate control group (Untrained Condition). Participants in the Untrained Condition received only general information about FP (this was designed to balance the exposure to information about FP in general between the two groups), and underwent a brief practice phase in identifying emotions expressed through facial expression (this was designed to balance the amount of time spent making judgments about emotions between the two groups).

The tasks which we administered consisted of questions about brief videos of individuals with FP. In the first phase of the experiment, the questions were designed to test participants' accuracy in identifying the emotions being expressed in the videos, as well as intensity of the emotions they attributed to the individuals in the videos, and also to probe what cues they relied upon in making these judgments. In the second phase of the experiment, participants were again presented with a video of an individual with FP describing his 
experiences. Now, however, they were asked questions probing how well they had been paying attention and were able to recall the content of what the individual had said.

We predicted that participants in the Trained Condition would be more accurate in identifying the emotions expressed in the videos in phase 1, that they would attribute a greater intensity of emotions to the individuals in the videos, and that they would be more likely to refer to non-facial cues in indicating how they had perceived the emotions in the videos. We also predicted that in phase 2, participants in the Trained Condition would be more accurate in responding to the questions probing their ability to recall the content of what the individuals with FP had recounted in the videos.

The hypotheses, sample sizes, methods, and initial analyses were all pre-registered before data collection. The pre-registration can be accessed at: https://aspredicted.org/blind.php? $\mathrm{x}=\mathrm{vw} 7 \mathrm{ih} 4$

\section{Study 1}

\section{Participants}

We determined that we would recruit 500 participants (without replacement), with random allocation to the two groups. 609 participants were recruited via Prolific Academic to take part in the survey in English. Participants were excluded if they did not give consent, self-reported as being under 18, did not complete the experiment, failed more than one of the control questions or if the time they took to complete the survey was not within 2.5 standard deviations of the mean completion time $(\mathrm{M}=1832, \mathrm{SD}=1018)$. After excluding 143 participants, the dataset included 466 participants (252 men, 211 women, 2 other, 1 prefer not to say). 256 participants identified their age as being between 18 to 29 (54.9\%), 110 between 30 to 39 (23.6\%), 51 between 40 to $49(10.9 \%), 32$ between 50 to $59(6.9 \%)$ and 7 above $60(3.6 \%)$. The majority of them $(74.9 \%)$ were caucasians. 128 of them $(27.5 \%)$ reported as working in other professions, $109(23.4 \%)$ being unemployed, $93(20 \%)$ in education, $67(14.4 \%)$ in business/human resources, 37 (7.9\%) in arts/entertainment/recreation and $32(6.9 \%)$ in health care/social assistance.

The experiment was conducted in accordance with the Declaration of Helsinki and was approved by the Humanities \& Social Sciences Research Ethics Sub-committee (HSSREC) at the University of Warwick. Participants were paid a $£ 2$ for their participation. 


\section{Stimuli}

\section{Familiarisation Stimuli}

For use in the familiarisation phase (see below), a naive actor who did not have FP was instructed to pose using facial expressions or body movements to represent 6 targeted emotions (anger, disgust, fear, happiness, surprise and sadness). The actor had given her informed written consent. The actor's expressions were then edited into 12 counterbalanced images ( 6 facial images for the Control Group and 6 body images for the Trained Group).

\section{Test Stimuli 1}

For use in test phase 1, one female (American accent) and one male (British Accent) with MS were instructed to recall past events to elicit targeted emotions which they described in their own words. Both had given their informed written consent. Their responses were filmed and edited into 6 thin slice clips $(M=20.8 s)$ which were shown to both groups in this order: female sadness, male happiness, female anger, male anger, female happiness, male sadness.

Video 1 (sad) lasted 24 seconds; it showed a woman with MS talking about being alone at home.

Videos 2 (happy) lasted 23 seconds; it depicted a man with MS describing his happy feeling when he first met his wife.

Video 3 (angry) video lasted 21 seconds; it showed a woman with MS commenting on difficulties with her partner.

Video 4 (angry) lasted 19 seconds; it showed a man with MS describing his anger while in Video 5 (happy) lasted 22 seconds; it depicted a woman with MS expressing her happiness spending time with her extended family.

Video 6 (sad) lasted 16 seconds; it showed a man with MS talking about feeling sad when people didn't understand his speech.

\section{Test Stimuli 2}

For use in test phase 2, a male with MS recorded a 2.5 minute long video describing events in chronological order about how he first met his wife on Facebook, started dating, traveled to America to meet her family and finally got married. He had given his informed written consent. The description contained time, geographic and biographical details. This video was shown to both groups. 


\section{Procedure}

After giving their informed written consent, participants were randomly assigned to either the Trained or Control Group. In both groups, they first answered demographic questions, then completed a familiarisation phase, followed by two test phases and a post-test phase. The full set of questions is available in the appendix. The videos are available on the OSF project page: osf.io/gn59u.

\section{Familiarisation Phase}

In the familiarisation phase, Participants in both groups read a brief general statement about Möbius Syndrome (MS), the form of FP present in the individuals in the videos used in the experiment. Participants were provided with information about the frequency of the condition.

In the Trained Group, participants were instructed not to rely on the face for information when communicating with people with FP. Next, participants were asked to imagine how to express themselves in a dating scenario if they had no facial expressions. Participants were also informed about typical symptoms of MS including speech and facial expression difficulties which affect movements in eyebrows, eyes and lips. To encourage proactive thinking, information from the training was edited into a multiple choice question to highlight the importance of compensatory communicative channels of people with FP. Generic feedback was given to participants after they answered the multiple choice question to reinforce their understanding. After feedback, participants viewed familiarisation stimuli ( 6 body images) and were asked to identify associated emotions in a single-choice format.

In the Control Group, participants were not prompted to pay attention to compensatory strategies. Instead, participants had the task of imagining how they would use facial expressions to communicate in a dating scenario. Participants were then tested on their understanding of the general statement given at the beginning of the familiarization phase in a multiple-choice format, followed by generic feedback. Finally, they were presented with familiasation stimuli ( 6 facial images) and selected the emotions they deemed as best-fit to the images.

\section{Test Phase I}


Both groups viewed Test Stimuli 1 in the same order (i.e. the order presented above, videos 16). After each of the six videos, participants were asked the following questions:

Accuracy Question: In order to prove their accuracy in identifying emotions, participants were asked to identify the actor's emotion from a list of the following options: fear, anger, sadness, surprise, happiness, disgust.

Intensity Question: In order to probe to what degree participants were sensitive to the strength of emotional expression, irrespective of the classification of the emotion, participants were asked to rate the intensity of the perceived emotion on a five-point scale ranging from 'not at all intense' to 'highly intense'.

Strategy Question: In order to probe to what extent participants relied on each of several different sources of emotion about the observed actors' emotions, participants were asked to rate to what extent they based their decision on: body language, gesture, tone of voice, words, the actor's face.

\section{Test Phase II}

After viewing the Test Stimuli 2 video for test phase 2, participants were presented with seven questions probing their recollection of the content of what the actor had said. The full list of questions can be found in the Appendix.

\section{Post-Test phase}

After the test phase, participants were asked the following control questions in order to enable us to identify and exclude bots as well as participants who had not been paying attention:

Control Question 1:

Was the sound turned on during the survey?

- Yes

- No

Control Question 2:

What was in the videos you watched?

- People talking about their experience.

- Animal foraging for food in the forest. 
- Children learning in a classroom.

- Seaside scenery

Control Question 3:

How many dogs did you see in the videos?

- None

- One

- Two

- Three

Control Question 4:

In the videos, where were the people sitting?

- In a room

- At the seaside

- In a forest

- In a restaurant

Finally, participants were asked to provide open-ended feedback about how useful they thought this study would be for them in interacting in the future with people with FP.

\section{Results}

Prior to the analyses, we excluded 132 participants who did not complete the experiment, 3 participants who failed more than one of the control questions, and 7 participants whose duration in completing the survey was more or less than 2.5 SDs different from the mean time $(\mathrm{M}=1832$ seconds, $\mathrm{SD}=1018)$ to complete the survey..

\section{Emotion recognition}

In order to investigate how the training affected participants' ability to accurately recognize an emotion expressed by someone with FP we carried out an independent samples t-test on accuracy scores between the trained and untrained group. Accuracy scores were determined for each participant by calculating that participant's percentage of correct responses to the six 
emotion recognition questions. This analysis did not reveal any significant difference between the two groups, $\mathrm{t}(464)=-.96, \mathrm{p}=.34, \mathrm{~d}=.09$.

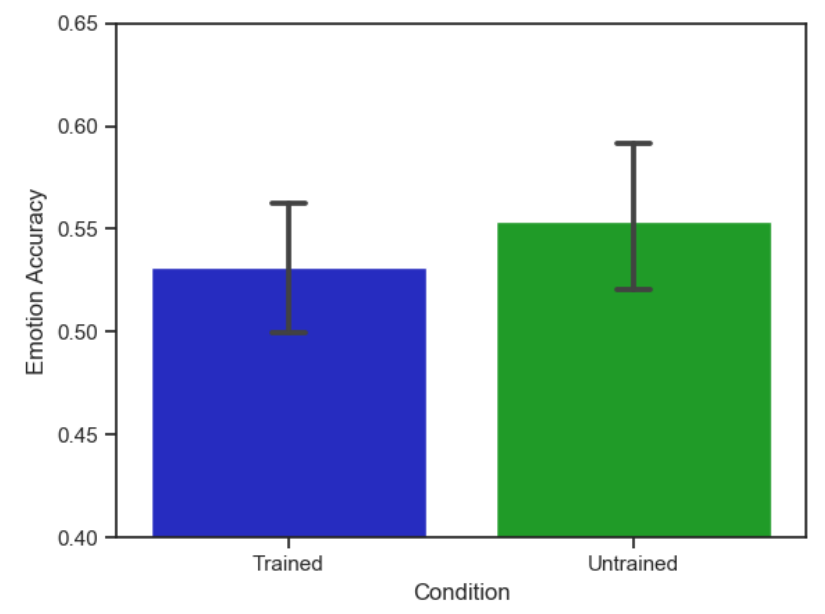

The extent to which the training affected participants' ability to recognize each of the three specific emotions (sadness, happiness and anger) expressed in the videos was investigated using three independent samples t-tests. For sadness participants in the untrained group were significantly more accurate than participants in the trained group. $\mathrm{t}(464)=-2.62, \mathrm{p}=.009, \mathrm{~d}=.25$. However there were no differences for happiness, $\mathrm{t}(464)=.59, \mathrm{p}=.55, \mathrm{~d}=.06$, or for anger, $\mathrm{t}(464)=-.06, \mathrm{p}=.94, \mathrm{~d}=.006$.

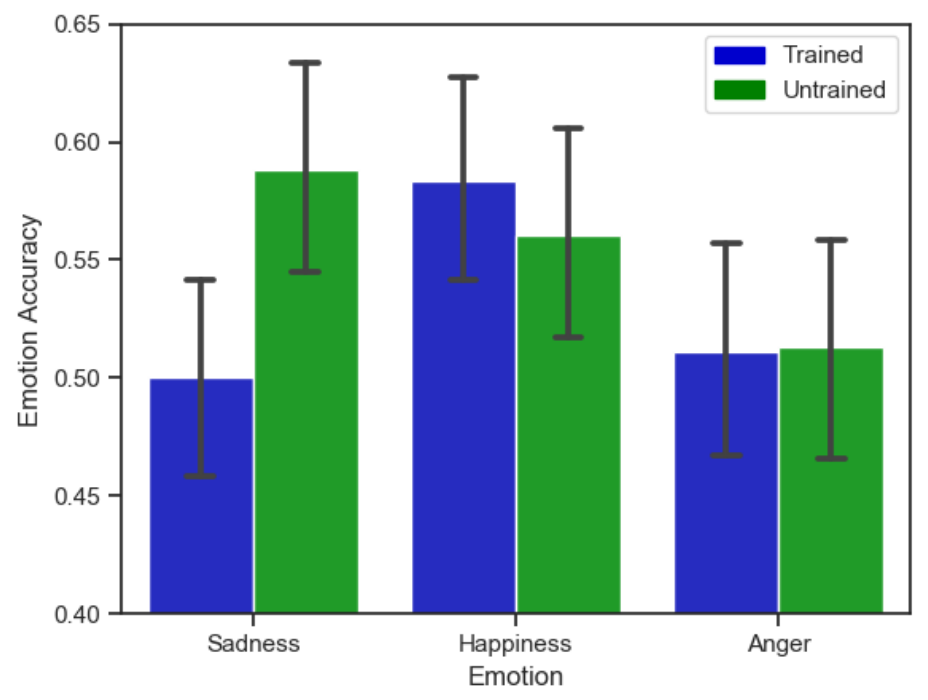




\section{Emotion intensity}

We also aimed to investigate how the training affected participants' perception of the intensity of the emotions expressed by those with FP. An independent t-test revealed that those in the trained group perceived the emotions as more intense than those in the untrained group $\mathrm{t}(464)=3.47, \mathrm{p}<.001, \mathrm{~d}=.32$.

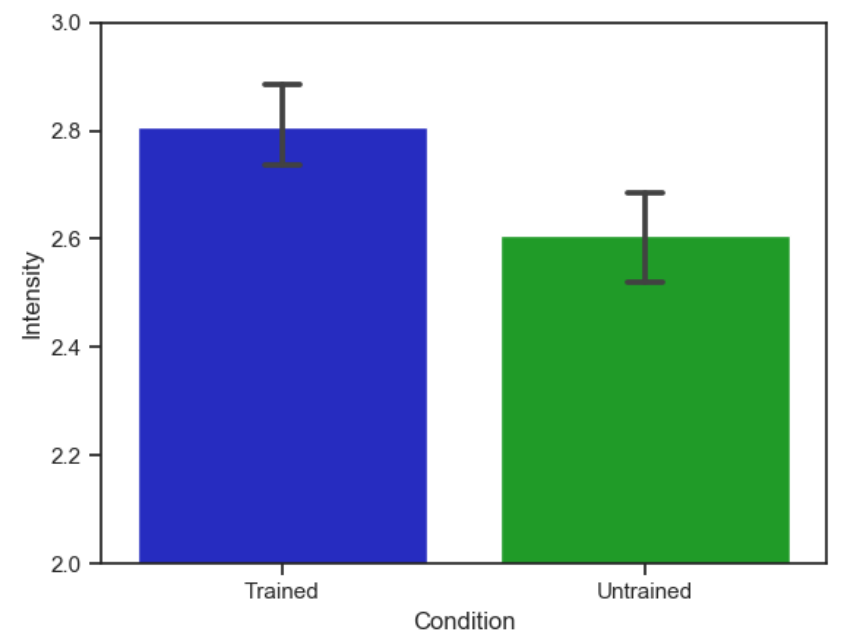

In order to ensure that participants were not biased towards rating the wrong emotions as more intense we carried out a linear mixed model with accuracy (correct, incorrect) and condition (trained, untrained) as factors. Instead of ANOVA we chose a linear mixed model as this analysis is less affected by large and unequal sample sizes. We found a significant main effect of accuracy $z=3.88, p<.001$, and condition, $z=2.69, p=.007$, but no interaction between accuracy and condition, $\mathrm{z}=-.71, \mathrm{p}=.48$. This suggests that the effect that training has on intensity is not simply down to a bias towards the wrong emotions. 


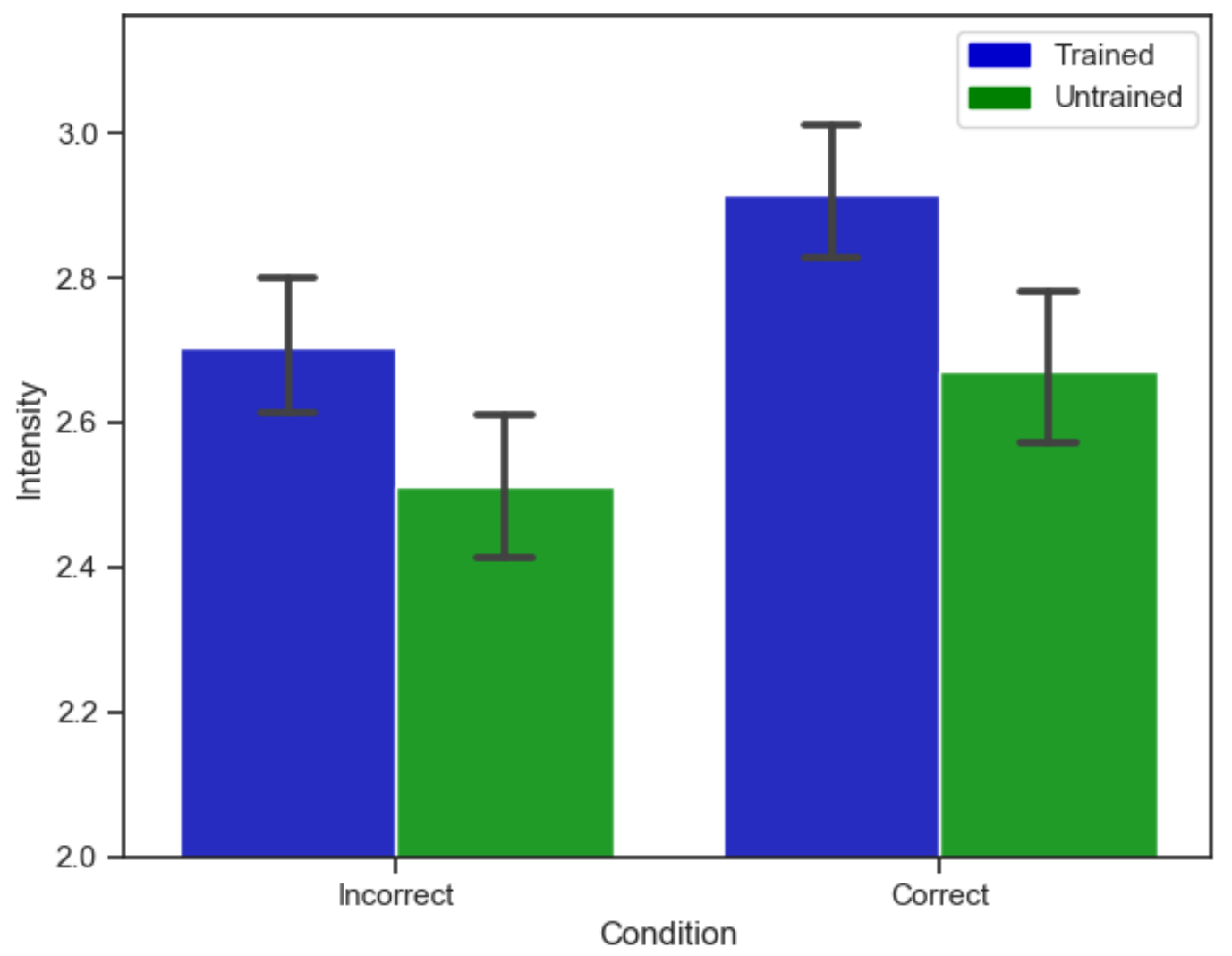

Across the three emotions, separate independent t-tests showed that trained participants rated emotions as more intense than untrained participants, for sadness, $\mathrm{t}(464)=2.86, \mathrm{p}=.007, \mathrm{~d}=.25$; for happiness, $\mathrm{t}(464)=2.61, \mathrm{p}=.009, \mathrm{~d}=.24$; and for anger, $\mathrm{t}(464)=3.02, \mathrm{p}=.002, \mathrm{~d}=.28$.

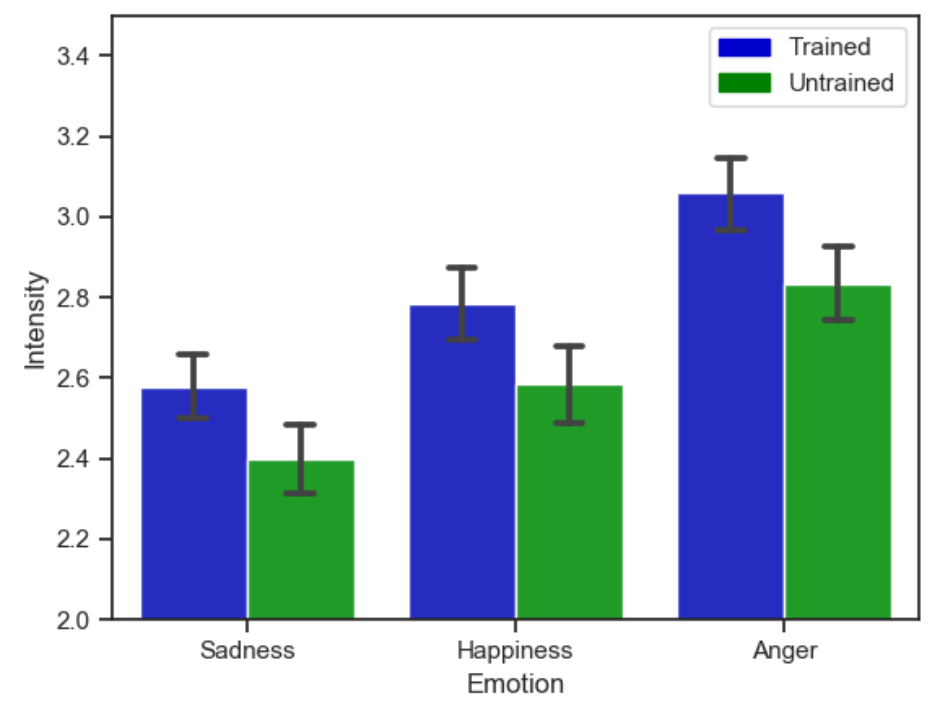




\section{Factual recognition}

As well as recognizing emotions, another aim of this study was to investigate how the training affected participants' ability to derive factual information from those with FP. We took participants' overall accuracy for the seven questions (\% correct) and conducted an independent samples t-test, $\mathrm{t}(464)=.12, p=.91, \mathrm{~d}=.01$, which revealed no differences between the trained and untrained group.

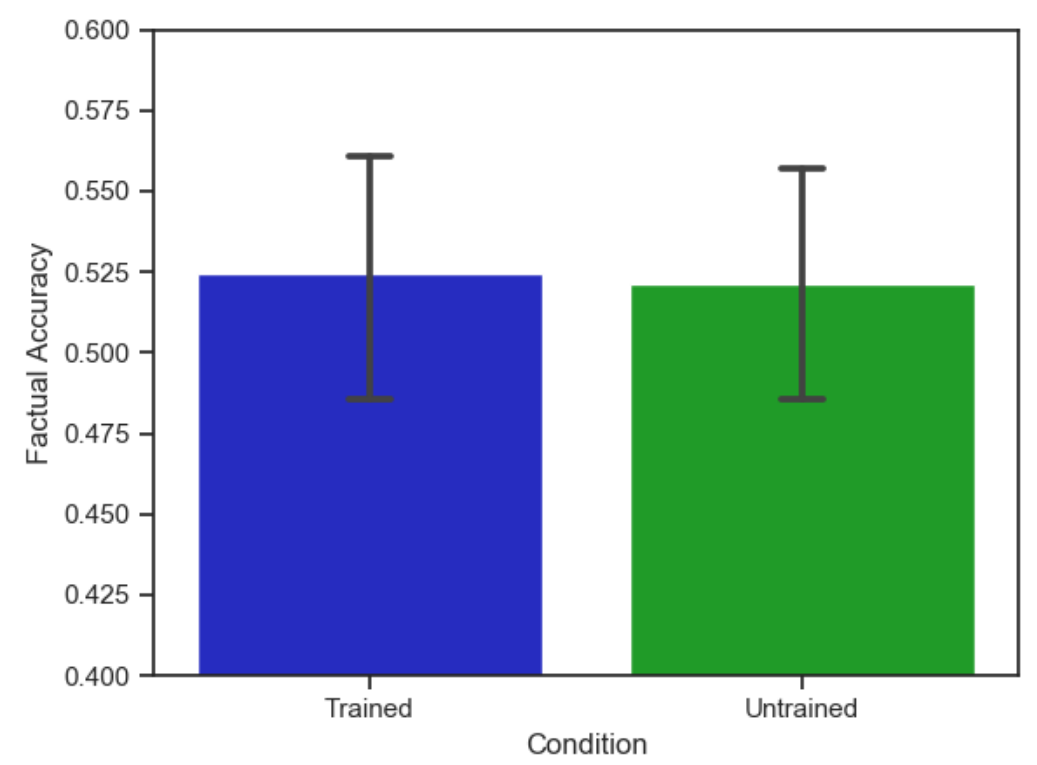

\section{Exploratory Analyses}

We also ran a battery of exploratory analyses on the data from this study.

The first set of analyses aimed to investigate to what extent participants relied on different cues to emotion (face, words, voice, gestures, and body language), and whether participants' responses regarding emotion (accuracy and intensity) and facts (accuracy) depended which of these cues they attended to. Because gesture and body language ratings were highly correlated, $\mathrm{r}=.77$, we collapsed them into one variable named bodily cues.

We found that trained participants attended to body cues significantly more than untrained participants, $\mathrm{t}(464)=3.25, \mathrm{p}=.001, \mathrm{~d}=.3$, and attended to the models face significantly less than untrained participants. $\mathrm{t}(464)=-4.72, \mathrm{p}<.001, \mathrm{~d}=.44$. However, trained participants did not attend to voice significantly more than untrained participants, $t(464)=1.92, p=.055$, $\mathrm{d}=.18$, and there was no difference between trained and untrained participants with regards to attention to words, $\mathrm{t}(464)=-.39, \mathrm{p}=.69, \mathrm{~d}=.04$ 

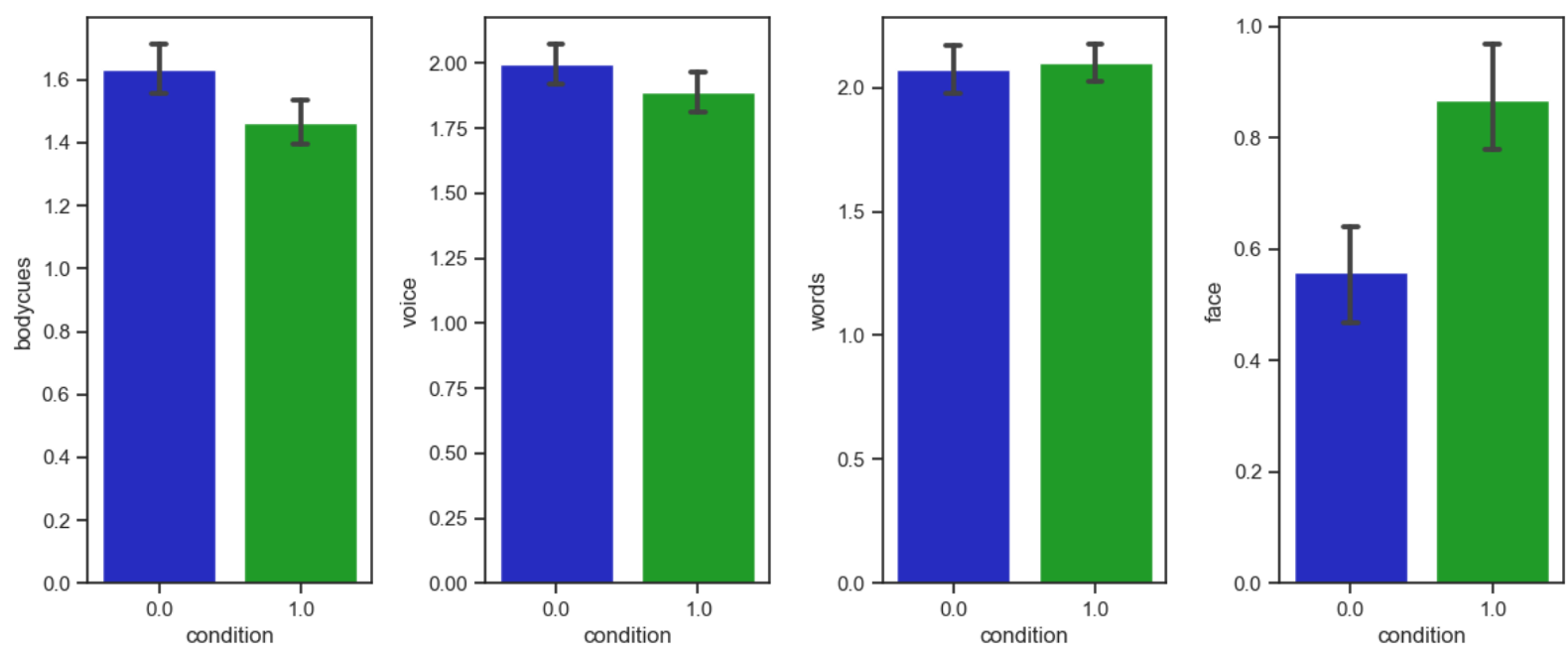

We then correlated participants' mean emotion accuracy, emotion intensity, and factual accuracy with the mean score for each of the ratings (indicating their overall tendency to attend to each of these cues).

Emotional accuracy was moderately positively correlated with attention to the models words, $\mathrm{r}(465)=.45, \mathrm{p}<.001$, and moderately negatively correlated with attention to the models face $r(465)=-.31, p<.001$. Moreover, emotional intensity ratings were moderately positively correlated with attention to bodily cues, $\mathrm{r}(465)=.38, \mathrm{p}<.001$, and voice, $\mathrm{r}(464)=.4, \mathrm{p}<.001$. There were also weak correlations for words, $r(465)=.23, \mathrm{p}<.001$ and face, $\mathrm{r}(464)=.21, \mathrm{p}<$ .001 . Finally, factual accuracy was moderately positively correlated to attention to the models words, $\mathrm{r}(465)=.39, \mathrm{p}<.001$, and moderately negatively correlated with attention to the models face, $\mathrm{r}(465)=-.28, \mathrm{p}<.001$.

Using Natural Language Processing (NLP) techniques, we explored the optional open ended responses that participants gave at the end of the study (378 out of 466), in order to get an idea of how the training affected how participants' felt about the task. Firstly, we split each participants sentence into single words (tokenization), removed any stopwords (words with no semantic content) and then transformed the remaining words into their respective root word (lemmatization). This process left us with 195 sentences (104 from the trained group and 91 from the trained group).

Firstly we plotted a frequency distribution for each group in order to identify common themes in the participants sentences. 


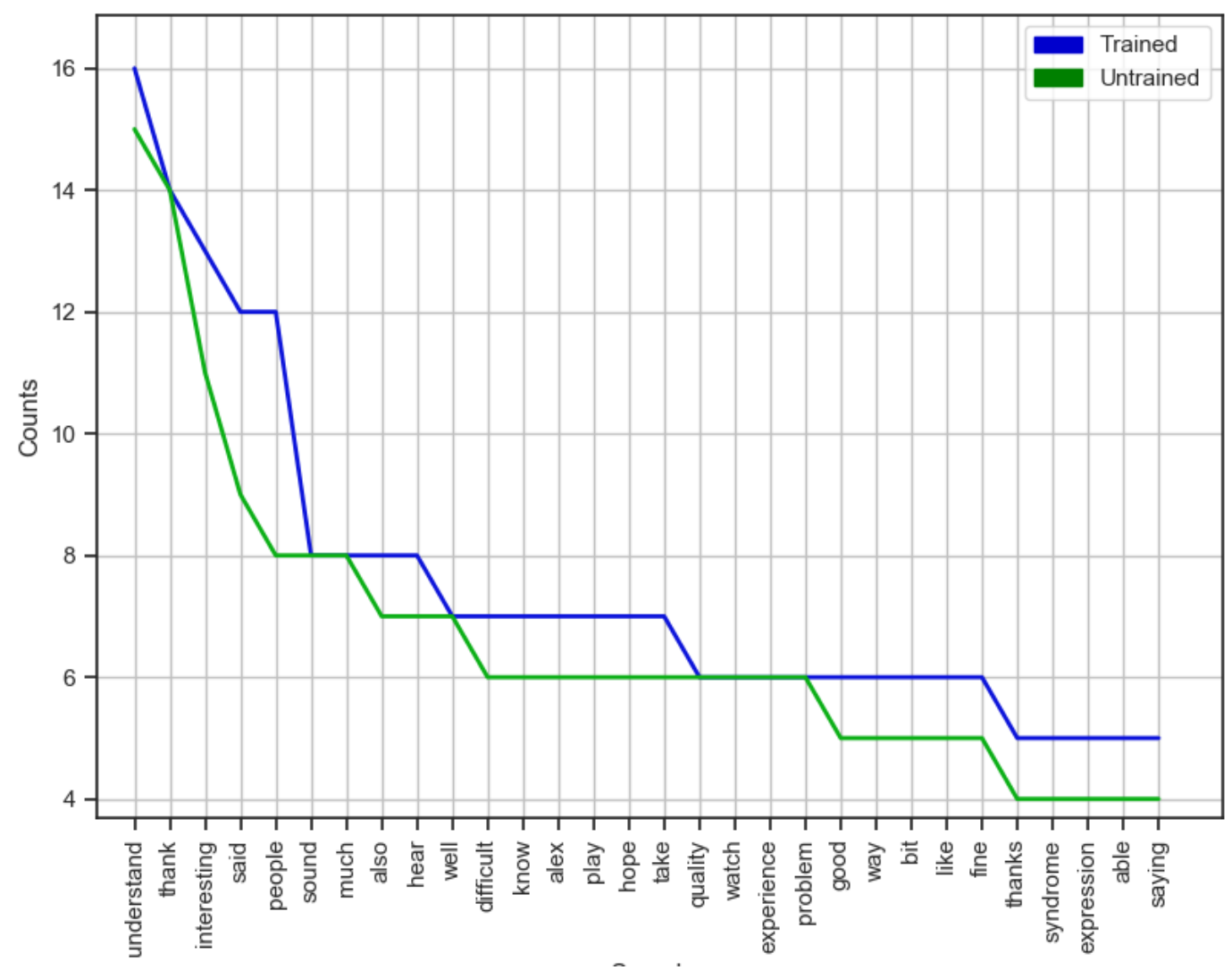

Secondly, we carried out a sentiment analysis on the sentences, assigning each sentence a value (sentence polarity) from -1 (negative) to +1 (positive) on the basis of the meaning of the words in that sentence. This gave us a crude measure of whether the participants had a more positive or negative attitude towards the task. Interestingly, an independent samples t-test comparing the sentiment scores between the two groups showed that trained participants responses were significantly more positive than the untrained participants responses, $\mathrm{t}(190)=2.78 . \mathrm{p}=.006, \mathrm{~d}=.4$. 


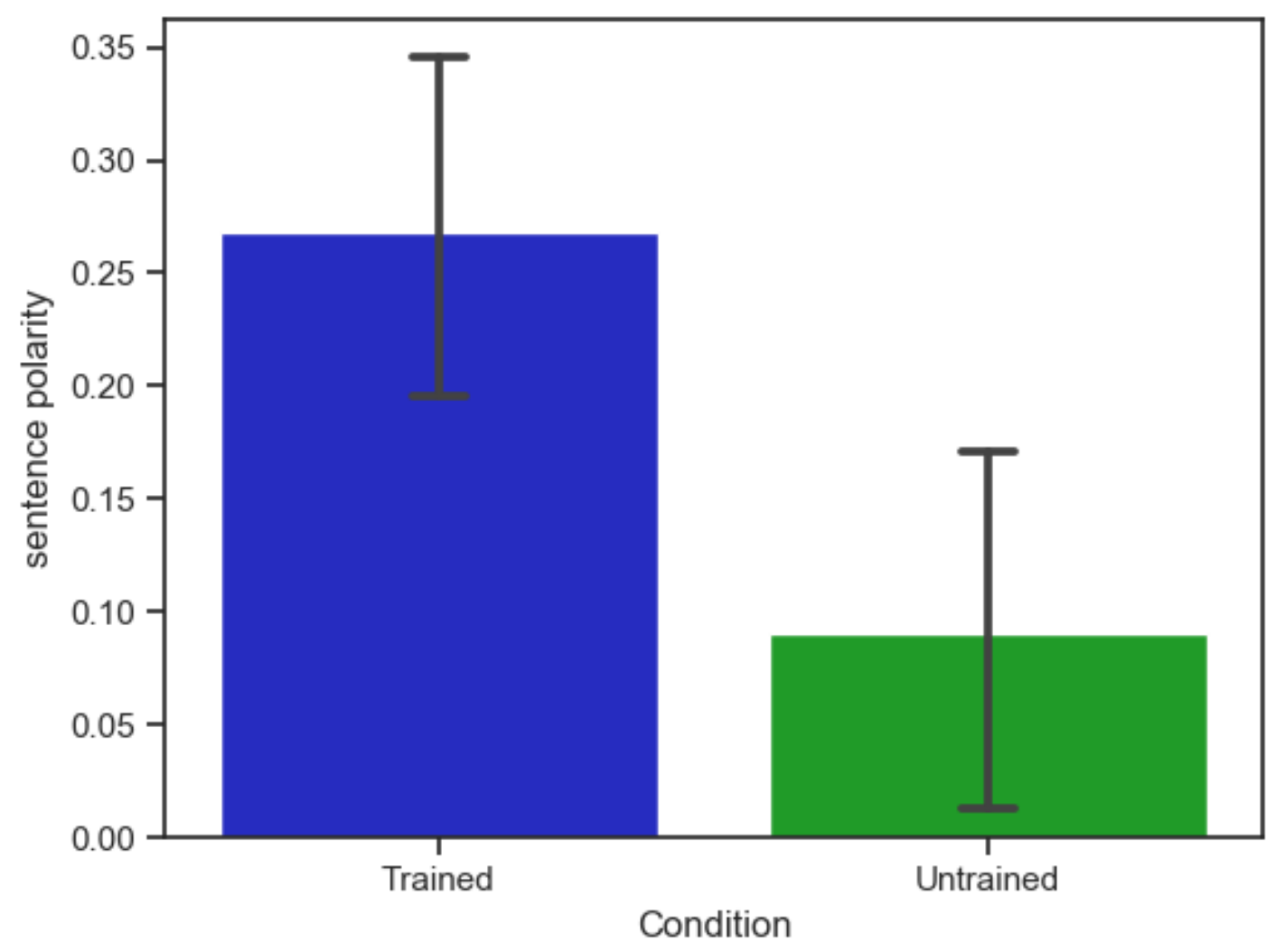

Study 2

In order to test the long term efficacy of the sensitivity training, we carried out a follow-up study one month after the initial study, in which those who had already completed the training were invited to complete the test phase again.

\section{Participants}

Approximately one month after the first study, we invited participants who had participated in the previous study to participate in the follow up. A total of 367 participants signed up through Prolific Academic to take part in the survey in English. Participants were excluded if they did 
not give consent, did not complete the experiment, failed more than one of the control questions or if the time they took to complete the survey was not within 2.5 standard deviations of the mean completion time $(\mathrm{M}=711 \mathrm{~s}, \mathrm{SD}=345 \mathrm{~s}) .180$ participants identified their age as being between 18 to 29 (52.9\%), 84 between 30 to 39 (24.7\%), 39 between 40 to $49(11.5 \%), 27$ between 50 to $59(7.9 \%)$ and 10 above $60(2.9 \%)$. The majority of them $(79.1 \%)$ were caucasians. 95 of them (27.9\%) reported as working in other professions, 84 (24.7\%) being unemployed, $61(17.9 \%)$ in education, $56(16.5 \%)$ in business/human resources, $25(7.4 \%)$ in arts/entertainment/recreation and $19(5.6 \%)$ in health care/social assistance. After excluding 27 participants, the dataset included 340 participants (183 men, 155 women, 2 other). See Table 1 for age, Table 2 for gender and Table 3 for professions. The experiment was conducted in accordance with the Declaration of Helsinki and was approved by the Humanities \& Social Sciences Research Ethics Sub-committee (HSSREC) at the University of Warwick. Participants were paid a $£ 1.20$ for their participation.

\section{Procedure}

The stimuli and procedure in Study 2 were the same as in Study 1, with the following exceptions. Most importantly, there was not familiarisation (i.e. training phase) at the beginning. We also eliminated test phase 2 in order to focus on performance in test phase 1 . Finally, we asked participants whether they recalled receiving tips for interacting with and perceiving individuals with FP. For those who responded that they did remember this, we also asked to what extent (on a 4-point scale from 'not at all' to 'all the time') those tips had been on their mind as they performed this follow-up study.

\section{Results}

\section{Emotion accuracy}

For emotion recognition accuracy, an independent samples t-test between those who recalled receiving training (Training Recall) and those who did not recall the training (No Training 
Recall) yielded a marginally significant difference in the predicted direction, $t(338)=1.91$, $\mathrm{p}=.057, \mathrm{~d}=.21$.

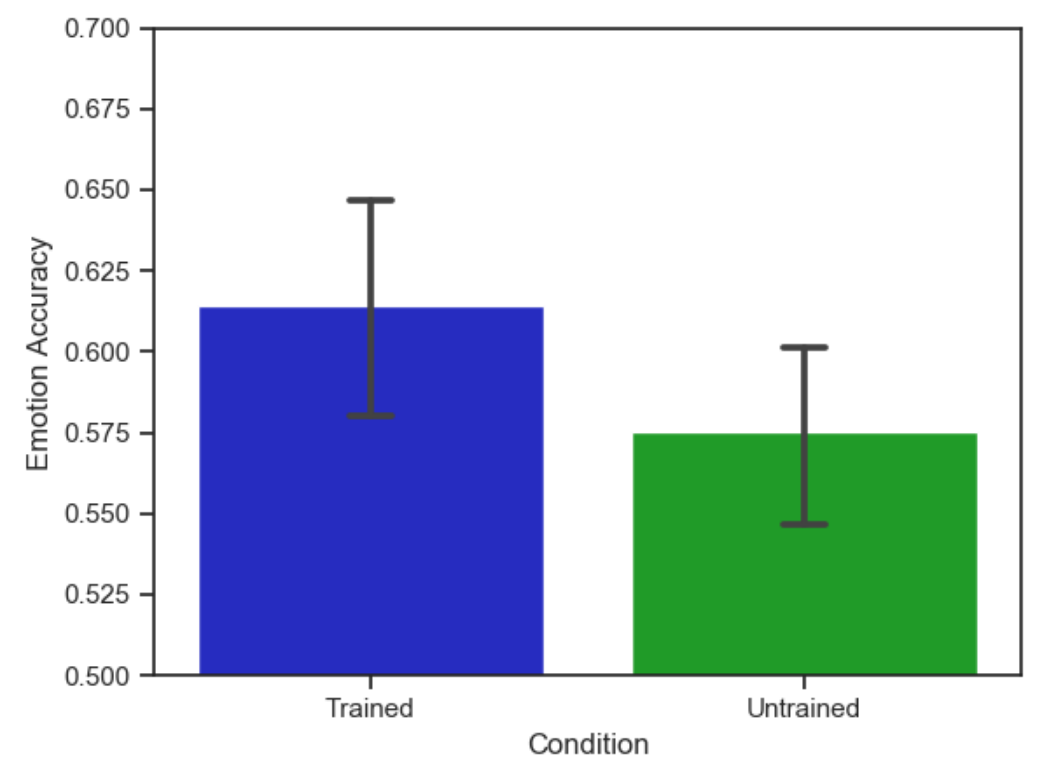

For emotion recognition accuracy across the three emotions, independent t-tests between trained and untrained participants yielded no significant differences for sadness, or anger, $\mathrm{t}(338)=.38, \mathrm{p}=.69, \mathrm{~d}=.05$, or anger, $\mathrm{t}(338)=1.05, \mathrm{p}=.29, \mathrm{~d}=.12$, and only a marginally significant difference for happiness, $\mathrm{t}(338)=1.93, \mathrm{p}=.054, \mathrm{~d}=.21$,

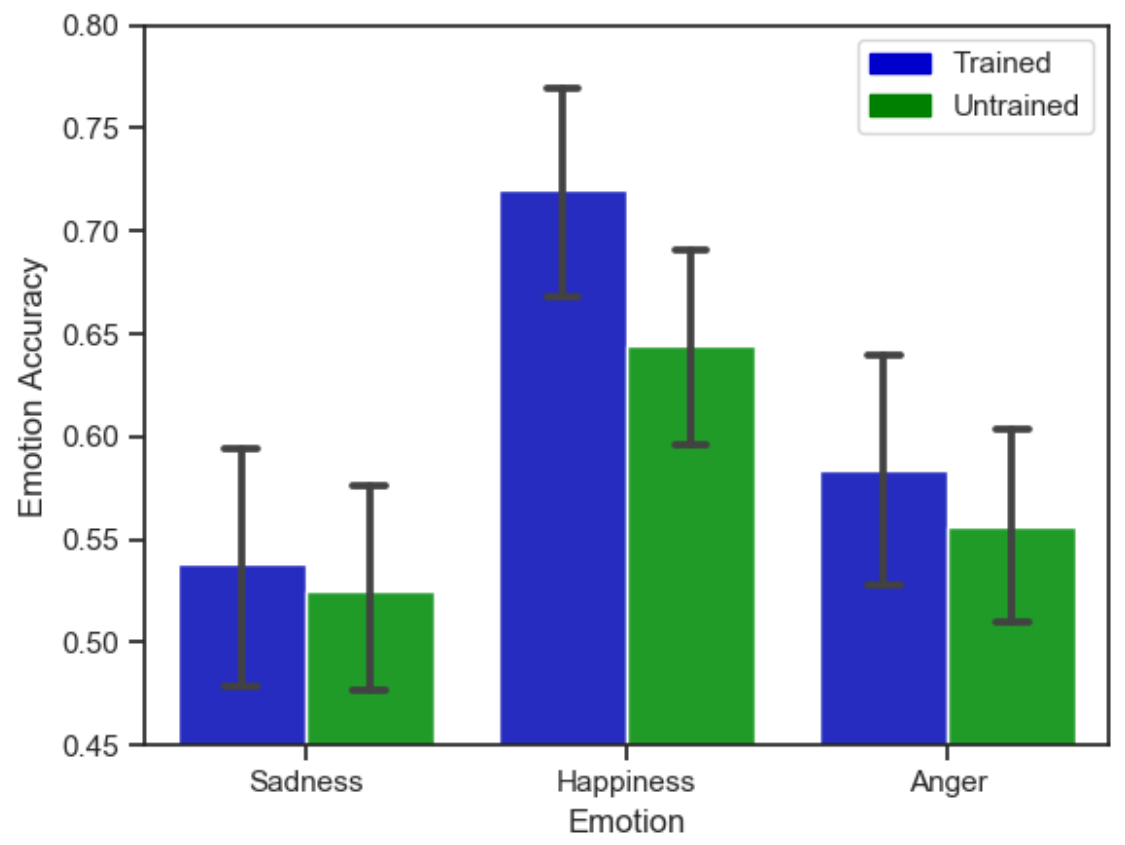




\section{Emotion intensity}

An independent t-test for emotion intensity ratings yielded no significant differences between those who were trained and those who were untrained, $\mathrm{t}(338)=.52, \mathrm{p}=.6, \mathrm{~d}=.057$.

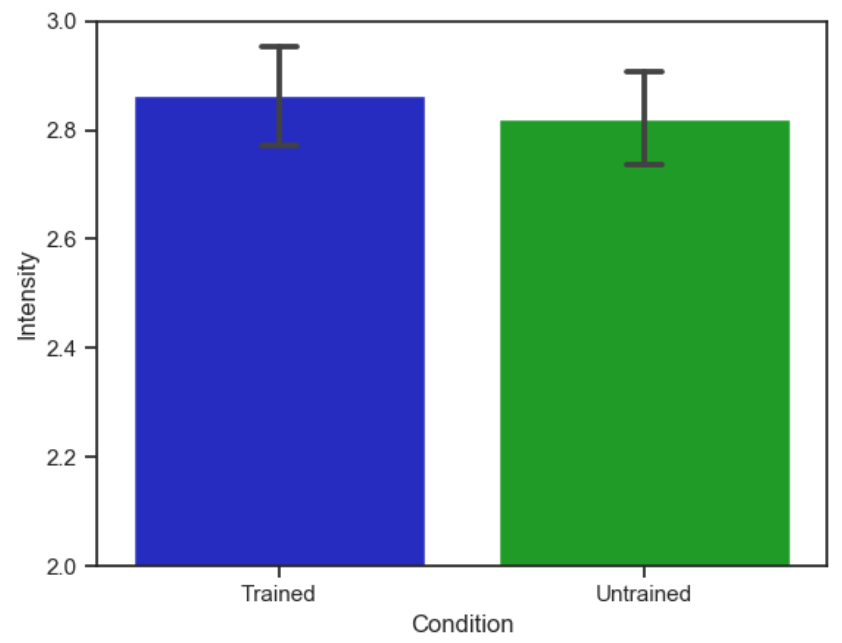

There were no significant differences regarding intensity ratings between trained and untrained participants, $\mathrm{t}(338)=.52, \mathrm{p}=.6, \mathrm{~d}=.08$, happiness, $\mathrm{t}(338)=.07, \mathrm{p}=.99, \mathrm{~d}=.001, \quad$ or anger, $\mathrm{t}(338)=.78, \mathrm{p}=.44, \mathrm{~d}=.09$.

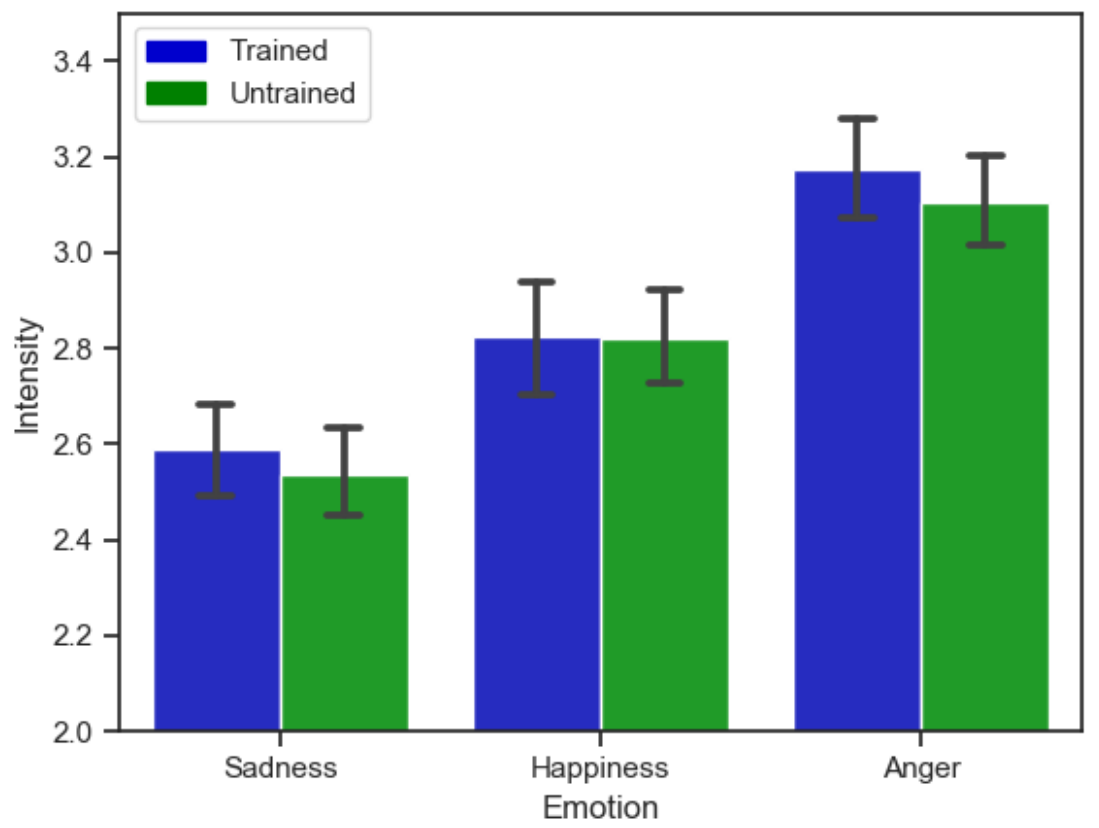

Exploratory Analyses 
As with study 1, we investigated whether or not the participants recalled the training had an effect on what cues they attended to when watching the video. Between conditions, there was no significant difference regarding attention to body cues, $\mathrm{t}(338)=.78, \mathrm{p}=.43, \mathrm{~d}=.08$, voice, $\mathrm{t}(338)=.24, \mathrm{p}=.81, \mathrm{~d}=.02$, or words, $\mathrm{t}(338)=1.79, \mathrm{p}=.07, \mathrm{~d}=.2$. However, participants in the Training Recall group attended to the model's face significantly less than the Training No Recall group, $\mathrm{t}(338)=-2.4, \mathrm{p}=.016, \mathrm{~d}=.26$.

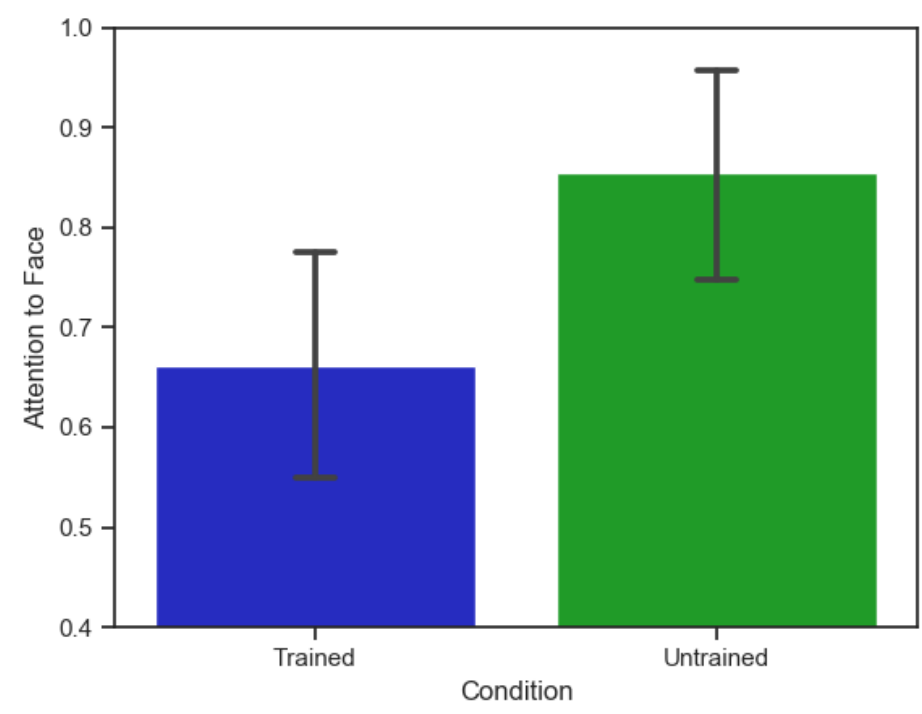

We also correlated mean emotional accuracy scores and mean emotional intensity ratings with participants tendency to attend to the models face, words, voice and body (again we collapsed gesture and body language due to high correlation between these two variables, $r$ $=.74)$.

For emotional accuracy, we found a moderate positive correlation between accuracy and attention to the models' words, $\mathrm{r}(338)=.42, \mathrm{p}<.001$, and a weak but significant negative correlation between accuracy and attention to the models' faces, $r(338)=-.21, p=.005$.

For emotional intensity we found weak positive correlations between perceived intensity and attention to body cues $\mathrm{r}(338)=.29, \mathrm{p}<.001$, voice, $\mathrm{r}(338)=.28, \mathrm{p}<.001$, and words, $\mathrm{r}(338)=.3, \mathrm{p}<.001$.

Like in Experiment 1, we used NLP techniques in order to gain insights from the openended sentences participants provided at the end of the study. After preprocessing (tokenization, lemmatization, removal of stopwords) we ended up with 89 sentences. We plotted word frequency, and then conducted a sentiment analysis on the words. The sentiment 
analysis yielded no significant differences between trained and untrained participants, with regards to sentence polarity, $\mathrm{t}(88)=.75, \mathrm{p}=.45, \mathrm{~d}=.16$.
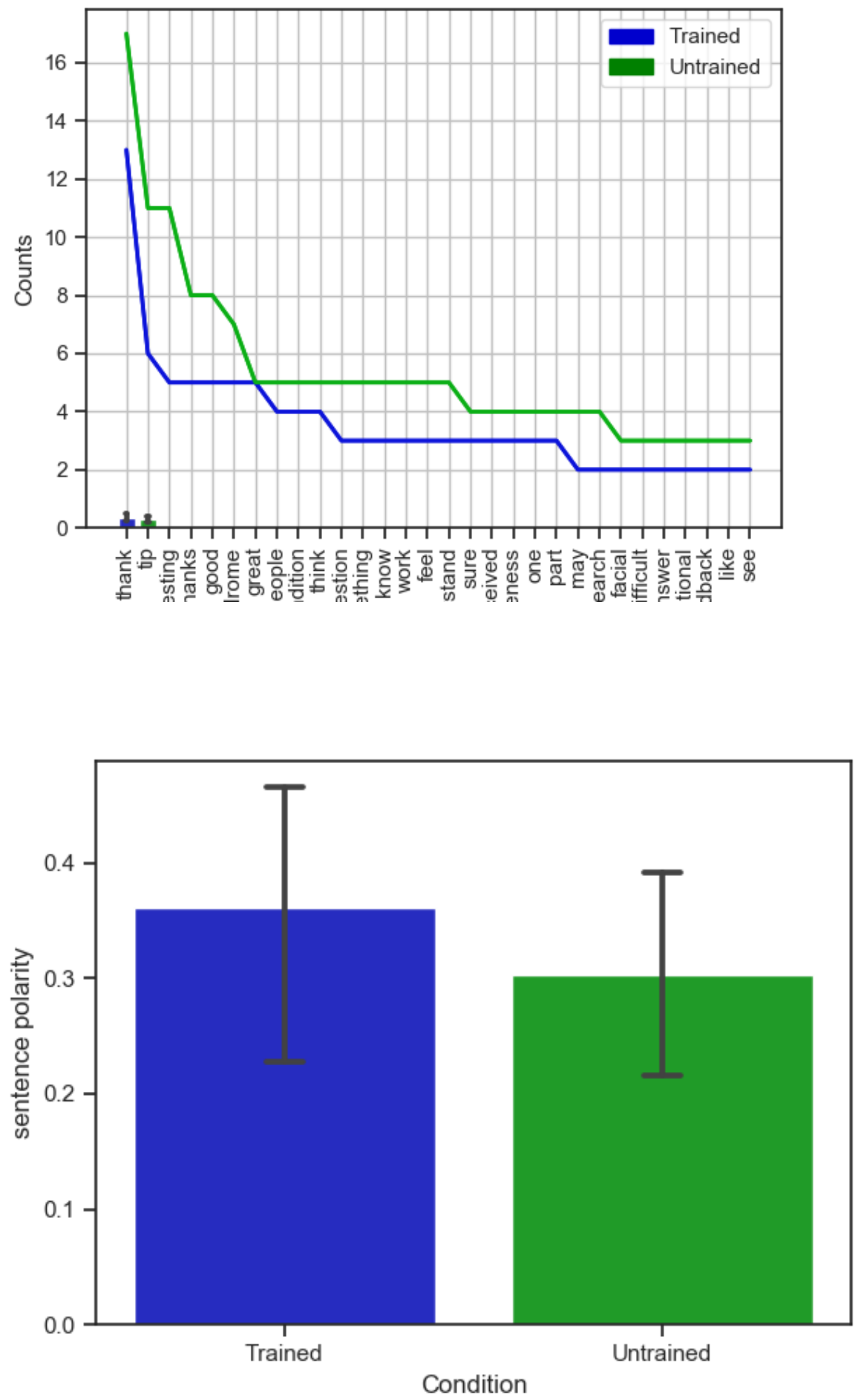

Discussion 
Across two studies, we tested the efficacy of a web-based training program which encouraged participants (in the Training Group) to attend to the gestures, body language and speech of individuals with FP rather than to the face. Contrary to our hypothesis, the results from both studies showed that participants in the Trained group were not more accurate at emotion recognition than participants in the Untrained group. In fact, participants in the Untrained group in Study 1 were more accurate than participants in the Trained group at recognizing sadness -this may reflect a bias towards perceiving people with FP as being sad.

The results for perceiving emotional intensity in Study 1, however, do support our hypothesis: participants in the Trained group in general perceived the individuals in the videos as exhibiting a higher degree of emotional intensity. This suggests that, though the training did not improve their ability to categorise perceived emotions, it may have made them more sensitive to emotional expression per se -- perhaps by reducing a prior bias to perceive people with FP as emotionally flat or disinterested.

For Phase 2 in Study 1, we predicted that the training would facilitate participants' ability to pay attention to and recall the content of what the individual in the video was recounting. The results, however, show no significant difference between conditions. It may be that participants who have just received the training are impaired by the cognitive load arising from the need to remember and apply what they have just learned. If so, then we might expect that people would perform better if they were prevented from viewing the faces of the individuals in the videos (e.g. if the faces were blocked) than if instructed to deliberately avoid looking at the faces. Indeed, we should expect that blocking out the faces of individuals with FP in videos would improve observers' accuracy, whereas blocking out the faces of individuals without FP would impair observers' accuracy.

The exploratory analyses revealed several interesting findings. Firstly, we observed in both studies that emotional accuracy was moderately positively correlated with attention to the models words, and moderately negatively correlated with attention to the models face. This is consistent with what our hypothesis should lead us to expect: paying more attention to what a person with FP is saying and less attention to their face is beneficial with respect to accurately perceiving their emotional state. Secondly, participants in both studies who paid more attention to bodily cues and to the tone of voice perceived a higher degree of emotional intensity than participants who paid less attention to these sources of information. Again, this is what our hypothesis would lead us to expect. Thirdly, accuracy in recalling what the individual had said was moderately positively correlated to attention to the words spoken and moderately negatively correlated with attention to the face. This, too, is consistent with our hypothesis. 
The results of these exploratory analyses suggest that encouraging people to attend to bodily cues and speech (content and tone of voice) may indeed improve accuracy at emotion recognition and help people to pay attention to and recall speech content, but that our training may not have been sufficiently extensive to produce clear effects.

We also found that participants in the Trained group in Study 1 reported attending more to bodily cues and speech, and less attention to faces, compared to participants in the Untrained Group. This finding suggests that our participants indeed implemented the training instructions.

Finally, exploratory analyses also revealed that participants in the Trained Group of Study 1 used more positively valenced words in the open-ended feedback questions at the end. We may speculate that this reflects either a more positive impression of the people in the videos they had viewed, a more positive experience viewing the videos, or both.

Taken together, our findings support the hypothesis that even brief training in attending to non-facial cues when interacting with individuals with FP can improve social perception and reduce bias, but that these effects do not persist over longer time periods in the absence of further training. Further research should attempt to design more intensive training, ideally involving interaction with people with FP rather than passive observation.

\section{References}

Bogart KR, Tickle-Degnen L, Ambady N. Communicating without the face: Holistic perception of emotions of people with facial paralysis. Basic Appl Soc Psychol 2014;36:30920.

Bogart, K. R., \& Tickle-Degnen, L. (2015). Looking beyond the face: a training to improve perceivers' impressions of people with facial paralysis. Patient education and counseling, 98(2), 251-256.

Hemmesch AR, Tickle-Degnen L, Zebrowitz LA. The influence of facial masking and sex on older adults' impressions of individuals with Parkinson's disease. Psychol Aging 2009;24:5429. 
Krueger, J., \& Michael, J. (2012). Gestural coupling and social cognition: Möbius Syndrome as a case study. Frontiers in human neuroscience, 6, 81 .

Lyons, K.D., L. Tickle-Degnen, A. Henry, and E. Cohn. 2004. "Impressions of Personality in Parkinson's Disease: Can Rehabilitation Practitioners See Beyond the Symptoms?." Rehabilitation Psychology 49 (4): 328.

Michael, J., Bogart, K., Tylén, K., Krueger, J., Bech, M., Østergaard, J. R., \& Fusaroli, R. (2015). Training in compensatory strategies enhances rapport in interactions involving people with Möbius syndrome. Frontiers in neurology, 6, 213.

Tickle-Degnen L, Lyons KD. Practitioners' impressions of patients with Parkinson's disease: the social ecology of the expressive mask. Soc Sci Med 2004;58:603-14.

\section{Appendix}

A: Familiarisation Questions

B. Test Phase II Questions

1. What did Alex think about Erin's family?

that they were amazing.

that they were hard-working.

that they were intelligent. 
- that it was a big family.

- I don't know.

2. What did Erin's dad suggest to Alex?

- that Alex go to the US.

- that Erin go to the UK.

- that He and Alex go to California together.

- that Alex could Skype with him.

- I don't know.

3. Why did Erin's dad ask Alex to return to the US?

- to surprise Erin.

. to help on the farm.

- to see his friends and extended family.

- to go to a conference.

- I don't know.

4. What did Alex do in the US?

- He proposed to Erin.

- He looked for a job.

- He went to a conference in New York.

- He helped Erin's dad on the farm.

- I don't know.

5. How often had Alex been to California?

- A few times

- Once

- Often

- $\quad$ Never

- I don't know.

6. Which holiday did Alex and Erin spend together after he arrived in the US?

- Valentine's Day

· Halloween

- Christmas 
- $\quad$ Easter

- I don't know.

7. How did Erin's parents feel about their relationship?

- Supportive

- Indifferent

- They didn't know about it.

- $\quad$ Not supportive

- I don't know.

8. Where did they put their wedding footage?
Facebook
Instagram
Youtube
- $\quad$ Reddit
- I don't know. 